individuals with early $\mathrm{RA}^{3}$ ) . However, few studies have detected the radiographic damage in the whole upper extremity on the mechanical stress.

Objectives: To examine the relationship between mechanical stress and radiographic damage in RA, we evaluated the joint destruction of the dominant and non-dominant upper extremity.

Methods: The joint destruction of the upper extremity (shoulder, elbow, wrist, metacarpo-phalangeal, interphalangeal, proximal interphalangeal) in 295 patients with RA, who were from 25 years to 91 years (mean age 64 years, mean disease duration 183 months, $86 \%$ females and $97 \%$ right-handed), was assessed according to the eroded joint, which was defined as $>2$ by Larsen scores for radiographic damage. These were divided into the dominant and non-dominant upper extremity. The Wilcoxon signed-rank test was used to examine the difference between the eroded joint count $(\mathrm{EJC})$ in the dominant and the non-dominant upper extremity.

Results: The mean EJC in the dominant and the non-dominant upper extremity was 2.4 and 2.05 respectively. The EJC was significantly more in the dominant than the non-dominant upper extremity. And, in regards to every joint of upper extremity, the eroded joint rate was higher in the dominant than the non-dominant

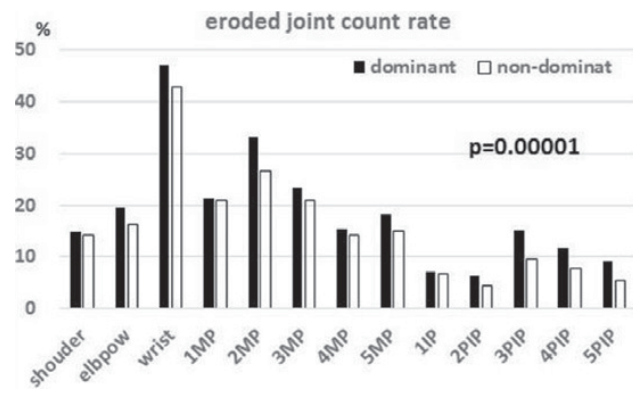

Conclusions: The eroded joint count of the upper extremity was significantly more in the dominant than the non-dominant one, therefore it was suggested that the mechanical stress influenced the radiographic damage in patients with $R A$ References:

[1] John $\mathrm{H}$ Bland, et al.: Hemiplegia and rheumatoid hemiarthritis. Arthritis Rheum (11) 1:72-80, 1968

[2] E N Glick, et al.: Asymmetrical rheumatoid arthritis after poliomyelitis. Br Med J 3: 26-28, 1967.

[3] Jung Hee Koh, et al.: Radiographic structural damage is worse in the dominant than the non-dominant hand in individuals with early rheumatoid arthritis. PLOS ONE, doi: 10. 1371/journal. Phone.0135409 August 6, 2015.

Disclosure of Interest: None declared

DOI: 10.1136/annrheumdis-2017-eular.3621

\section{FRI0167 COMPARATIVE CARDIOVASCULAR SAFETY OF ABATACEPT AND TUMOR NECROSIS FACTOR INHIBITORS IN RHEUMATOID ARTHRITIS PATIENTS WITH AND WITHOUT CARDIOVASCULAR DISEASE: A POPULATION-BASED COHORT STUDY}

Y. Jin ${ }^{1}$, E.H. Kang ${ }^{1,2}$, G. Brill ${ }^{1}$, R.J. Desai ${ }^{1}$, S.C. Kim ${ }^{1,3} \cdot{ }^{1}$ Division of Pharmacoepidemiology and Pharmacoeconomics, Brigham and Women's Hospital, Harvard Medical School, Boston, United States; ${ }^{2}$ Division of Rheumatology Department of Internal Medicine, Seoul National University Bundang Hospital, Seongnam, Korea, Republic Of; ${ }^{3}$ Division of Rheumatology, Immunology and Allergy, Brigham and Women's Hospital, Harvard Medical School, Boston, United States

Background: Rheumatoid arthritis (RA) patients have an increased risk of cardiovascular disease (CVD). Recent studies suggested that treatment of RA with tumor necrosis inhibitors (TNFi) can reduce the risk of cardiovascular events. However, it is unclear how abatacept, a selective costimulation modulator, affects cardiovascular risk among RA patients compared with TNFi.

Objectives: To evaluate the comparative cardiovascular safety of abatacept versus TNFi in RA patients with and without underlying CVD.

Methods: We identified RA patients aged $\geq 18$ with $\geq 2$ RA ICD-9 codes (714.xx) separated by $>7$ days but $<365$ days, from two large insurance claims data across the U.S.: Medicare (2008-2013) and Truven MarketScan (2006-2015). Only new users of abatacept or TNFi (adalimumab, etanercept, certolizumab, golimumab, and infliximab) were included. The primary outcome of interest was a composite endpoint of CVD including myocardial infarction (MI), stroke/transient ischemic stroke, or coronary revascularization. Secondary outcomes were each component of the primary outcome, incident heart failure (HF), and venous thromboembolism (VTE). 1:1 propensity score (PS) matching was performed separately in each database and each subgroups (with or without baseline CVD). Then the PS-matched subgroups were aggregated to form the overall matched cohort. Cox regression model was used to estimate the hazard ratio $(\mathrm{HR})$ and $95 \%$ confidence interval $(\mathrm{Cl})$ of risk of each outcome. Estimates from two different databases were combined through an inverse variance-weighted fixed-effects model.

Results: After 1:1 PS matching, there were 6,102 patient pairs from Medicare and 6.934 pairs from MarketScan. Among them, patients with baseline CVD were $35.3 \%$ in Medicare and $14.0 \%$ in MarketScan. Baseline characteristics were well balanced between two treatment groups after matching (standardized mean difference $<0.1$ ). In Medicare cohort, abatacept consistently showed a decreased risk of composite CVD compared with TNFi in overall and each subgroup (Figure). However, in MarketScan cohort, where the population was younger than Medicare cohort, there was no association between abatacept and CVD compared to TNFi. After combining the two databases, abatacept was significantly associated with reduced risk of composite CVD outcome vs. TNFi in overall cohort ( $\mathrm{HR}=0.79$, 95\% $\mathrm{Cl}=0.67-0.92$ ) and baseline CVD subgroup ( $\mathrm{HR}=0.79,95 \% \mathrm{Cl}=0.64-0.98$ ). We also observed similar trend for secondary outcomes, where abatacept had decreased risk than TNFi.

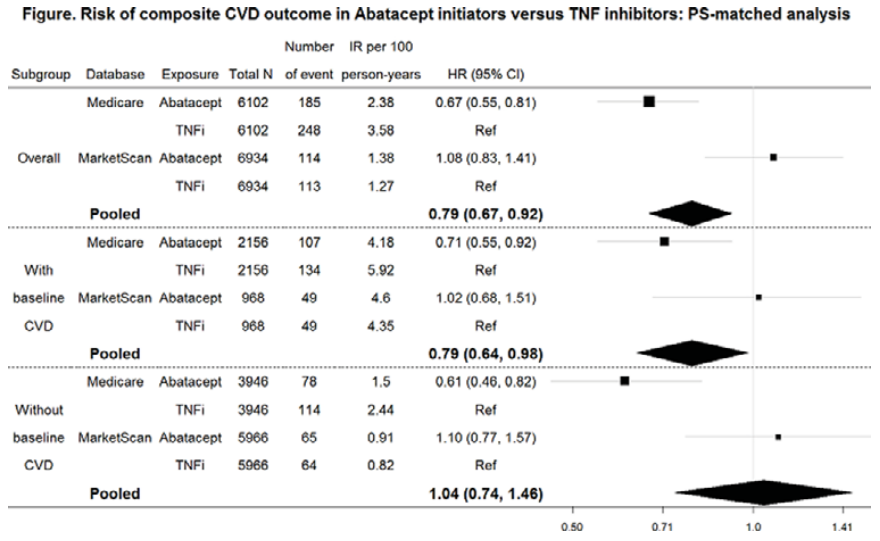

Conclusions: In this large multi-database population-based study of RA patients abatacept treatment was associated with reduced risk of CVD compared to TNFi, especially among older population and patients with prior CVD conditions.

Disclosure of Interest: None declared

DOI: 10.1136/annrheumdis-2017-eular.2727

\section{FRI0168 RESTORATION OF DECREASED LYMPHOCYTES, CD8+T CELL SUBSETS WITH TH1 SKEWED PHENOTYPE ASSOCIATE WITH SPONTANEOUS REGRESSION OF LYMPHO-PROLIFERATIVE DISORDERS IN RHEUMATOID ARTHRITIS PATIENTS TREATED WITH METHOTREXATE}

S. Saito ${ }^{1}$, K. Suzuki ${ }^{1}$, K. Yamaoka ${ }^{1}$, K. Amano ${ }^{2}$, M. Tokuhira ${ }^{3}$, T. Takeuchi ${ }^{1}$. ${ }^{1}$ Division of Rheumatology, Department of Internal Medicine, Keio University School of Medicine, Tokyo; ${ }^{2}$ Division of Rheumatology, Saitama Medical Center, Saitama Medical University; ${ }^{3}$ Division of Hematology, Saitama Medical Center, Saitama Medical University, Saitama, Japan

Background: Lympho-proliferative disorder (LPD) is known as a relatively rare but life-threatening complication in RA patients under MTX administration. Spontaneous regression of LPD after MTX withdrawal is regarded as a distinct character of LPD under MTX administration. Previous study from our institution [1] and others [2] reported the link between decreased lymphocyte counts at LPD diagnosis and restoration after MTX cessation and the regression of LPD.

Objectives: To investigate the immunological factors including lymphocyte sub sets which involved in spontaneous regression of LPD following MTX withdrawal. Methods: We studied 35 RA patients complicated with LPD under MTX administration in our institution. Age, sex, RA disease duration matched contro MTX-treated patients $(\mathrm{N}=35)$ were selected. LPD patients were divided into two groups regarding to the status of LPD after MTX cessation; regressive group $(\mathrm{N}=22)$ and persistent group $(\mathrm{N}=13)$. Clinical features were compared among 3 groups. Flowcytometric analysis of the whole blood sample and measurement of cytokine concentration in ELISA were conducted in a part of the LPD patients $(\mathrm{N}=10,7$ regressive, and 3 persistent LPD) and controls $(\mathrm{N}=10)$. The time of MTX cessation, which was simultaneous with LPD diagnosis, was defined as week 0 and blood sample was collected at week 0,4 and 12 .

Results: At week 0 , number of peripheral lymphocytes was significantly decreased in regressive group, compared to persistent group and control group. Flowcytometric analysis revealed significant decrease in proportion of effector memory CD8+ $T$ cells (EMCD8+T), Epstein Barr Virus specific CD8+ $T$ cells (EBV specific CD8+) and T helper 1 cells (Th1 cells) subset in regressive group compared to control group. Following MTX cessation, significant increase in proportion and absolute number of these subsets were observed only in the regressive group, but not in persistent group. Expansion of Th1 cells and EMCD8+ $\mathrm{T}$ cells significantly correlated with increase of serum IFN- $\gamma$, and expansion of EMCD8+ T cells inversely correlated with change of serum IL-15.

Conclusions: Restoration in proportion and absolute number of Th1 cells, EMCD8+T cells and EBV specific CD8+ T cells coincided with increase of IFN- $\gamma$ and associated with regression of LPD developed under MTX administration Since changes of those immunological factors were not observed in persistent LPD, this study would be the first report to demonstrate the difference of 